\title{
Pure Partial Awareness or Interaction between the Mask and the Masked Stimuli?
}

\author{
Jiangong Guo ${ }^{1 *}$, Chengzhen $\mathrm{Liu}^{1}{ }^{*}$, Jerwen Jou${ }^{2}$, Qian Cui ${ }^{3}$, Guang Zhao ${ }^{4}$, Shen $\mathrm{Tu}^{1}$ \\ ${ }^{1}$ Department of Psychology, Institute of Education, China West Normal University, Nanchong, China \\ ${ }^{2}$ Department of Psychology, University of Texas-Rio Grande Valley, Edinburg, TX, USA \\ ${ }^{3}$ School of Political Science and Public Administration, University of Electronic Science and Technology of China, \\ Chengdu, China \\ ${ }^{4}$ School of psychology, Liaoning Normal University, Dalian, China \\ Email: shentu614@yeah.net
}

Received 1 March 2016; accepted 29 May 2016; published 1 June 2016

Copyright (C) 2016 by authors and Scientific Research Publishing Inc.

This work is licensed under the Creative Commons Attribution International License (CC BY).

http://creativecommons.org/licenses/by/4.0/

c) (i) Open Access

\begin{abstract}
Many studies using masking paradigm have shown that stimuli can be processed unconsciously. However, different researchers put forward different ideas about the mechanisms of the unconscious information processing. For example, one idea is that the unconscious information might be derived from a partial awareness of the masked stimulus. Another idea is that it is derived from a perceptual interaction between a masked stimulus and the masking stimulus. We used a masking paradigm (with a briefly displayed target followed by a mask) and a subjective rating and an objective forced-choice test (with a word and picture version) given after the display to study the nature of partial awareness. The question we attempted to answer was whether people did perceive fragmentary features of a masked object picture correctly when they rated it as partially perceivable. The results showed that even when the masked stimuli only had simple features and when the subjects subjectively reported that they could perceive something of the masked stimuli, the objective forced-choice test performance was at chance level. The results were discussed in the context of interaction hypothesis and level of processing hypothesis.
\end{abstract}

\section{Keywords}

Masking, Unconscious Processing, Partial Awareness, Interaction Hypothesis, Level of Processing Hypothesis

\footnotetext{
"Jiangong Guo and Chengzhen Liu contributed equally to this work.
} 


\section{Introduction}

Many studies have demonstrated that information processing can take place at an unconscious level (Henson, Mouchlianitis, Matthews, \& Kouider, 2008; Montoro, Luna \& Ortells, 2014; Yun et al., 2011), which can affect subsequent responses (Arminjon et al., 2015; Faivre \& Kouider, 2011). In addition, the unconscious processing is found to be not necessarily automatic but susceptible to modulation by top-down attentional processes (Liu et al., 2015; Pinto, van Gaal, de Lange, Lamme, \& Seth, 2015). In studies on unconscious processing, masking is most commonly used as a means to keep the information processing unconscious. In this procedure, a briefly presented stimulus is followed immediately by a masking stimulus (Atas, Vermeiren, \& Cleeremans, 2013). To assess the awareness of the masked stimuli, both subjective report (e.g., verbal reports) and objective measures (e.g., performance on a forced-choice discrimination test) are used, often concurrently (Atas et al., 2013; Heeks \& Azzopardi, 2015; Vermeiren \& Cleeremans, 2012), but typically with the objective measure used more commonly.

Although many studies have observed an effect of a masked stimulus and usually considered it to reflect the unconscious processing of the masked stimuli, some researchers suggested other possible explanations for the effect. For example, Kouider and Dupoux (2004) proposed that the semantic priming effect was the result of the subjects' partial awareness of the masked stimulus. Partial awareness is a cognitive state between complete awareness and complete unawareness (Kouider, de Gardelle, Sackur, \& Dupoux, 2010; Kouider, de Gardelle, \& Dupoux, 2007). In a partial awareness state, an observer can be aware of some fragments or features of a stimulus, e.g., some letters of a word (Kouider \& Dupoux, 2004), or the contour of an object (Tu, Qiu, Martens, \& Zhang, 2013), but not the identities of the word or the stimulus. According to Block (2007), in a partial awareness state, the available information is quantitatively rich but qualitatively poor (Block, 2007). Recently, we found that the conscious component of partial awareness of serially-presented, masked object pictures (e.g., the contour information but not the identity of the object) could interact with the category-selective top-down modulation on the unconscious component of the same masked object pictures (Tu et al., 2013). The interaction effect in the middle occipital gyrus even seemed to be in a successive manner by controlling the pattern of alternation across five serially-presented masked object pictures, i.e., different tool pictures, mirror-image tool pictures and invariant tool pictures (Tu et al., 2015). Besides partial awareness of the masked stimulus, the possible perceptual interactions between the masked stimulus and the mask may also account for the unconscious priming effect in the masking paradigm (Sumner, 2007). For example, in some experiments, the mask contained some masked-stimulus features (e.g., the mask was constructed by superimposing the different masked stimuli " $<<$ " and “>>”) (Verleger, Jaśkowski, Aydemir, Lubbe, \& Groen, 2004). Sumner (2007) suggested that in this case the mask played a role in the generation of the negative compatibility effect. He even considered the maskedstimulus and mask sequence as one dynamic stimulus rather than two discrete, static ones.

Because of the complications caused by partial awareness and interaction between the masked stimulus and the mask, we believe that the objective measure needs to be improved to make sure that the supposedly unconscious information of the masked stimulus that the subjects may be using is indeed unconscious and other confusion (e.g. interaction between the masked stimulus and the mask) is not involved. Only then can we get a better understanding about the nature of unconscious information processing especially in the unconscious priming studies. Typically, an objective measure of unconscious information employs a forced-choice task in which subjects are asked to select/guess which one of the given alternatives is the masked stimulus. In some cases, the choices in the forced-choice discrimination task are from the same category (e.g. two faces), and both choices have similar characteristics to the masked stimulus. Therefore, in those cases, it is unclear whether a performance at chance level reflects complete unconsciousness of the masked stimulus or an impossibility to discriminate the details of the two alternative stimuli from the same category of the masked stimulus (Henson et al., 2008). In the study of Tu et al. (2013) noted above, the two choices of the forced-choice task used two words denoting two different objects one of which was the masked object. The results indicated that the subjects could not recognize the masked object picture presented before the test. By combining the results from the objective test with those from the subjective reports (in which subjects reported sensed contours of the objects), Tu et al. (2013) concluded that subjects were partially aware of the masked stimuli. However, in Tu et al.'s (2013) study, the contents of partial awareness were not investigated. Subjects just reported that they sensed some contours. Because of the possibility of an interaction between the object picture and the mask, which could bias or otherwise alter the perception of the masked stimuli, it was not clear whether subjects could indeed correctly perceive 
the characteristics of the contour of an object at a partial awareness level.

In the present study, we directly investigated whether the subjects can be aware of the contour of the tool objects at a partial awareness level by making the following modifications in design and procedure. First of all, the mask was adjusted to make the subjects partially aware of the masked stimuli. Then two versions of a forcedchoice task were used, a word version and a picture version. In the word version of the forced-choice task, two words were presented as two choices, one of which named the masked stimulus and the other named a different object. This version was intended to confirm our hypothesis that subjects could not recognize the identity of the masked stimuli at the partial awareness level. In the picture version of forced-choice task, two pictures were presented, one of which depicted the masked stimulus and the other was the mirror image of the masked stimulus. We hypothesize that if subjects were aware of the contour but not the identity of the masked object in Tu et al. (2013), then the performance of the word version of the forced-choice task should be at the chance level but the performance of picture version of the test should be above the chance. The hypothesis is based on the idea that the correctly perceived contour features can be used to make the correct responses in the picture version of the forced-choice task which did not require the knowledge of the identity of the object but will not be helpful in the word version of the forced-choice task which required the knowledge of the object's identity.

\section{Method}

\subsection{Subjects}

Thirty-seven subjects (21 women and 16 men, 19 to 21 years old) from China West Normal University participated in this experiment voluntarily. Each subject participated in only one version of the forced-choice task (18 subjects in the word version of the forced-choice task and 19 in the picture version of the task). All subjects had normal or corrected-to-normal vision and reported no history of neurological or psychiatric disorders. After the description of the task, they gave a written informed consent.

\subsection{Stimuli}

Fifty-five tool pictures were selected from the Internet as the stimuli. All pictures were transformed into grayscale images. Furthermore, all tool pictures were bilaterally asymmetrical to produce their mirror images that had different contours than the original images. As a result, the two versions of a picture (one being the mirror image of the other) had different orientations/shapes of the contour. Each picture subtended approximately 4.3 (height) $\times 3.8$ (width) degrees of visual angle.

Before the experiment, five subjects who did not participate in the formal experiment were asked to report whether they could sense some features of the masked stimuli. The procedure was similar to that of the formal experiment (see Figure 1 for the sequence of stimuli in the display), except that the forced-choice response was replaced by yes or no response (whether they could sense some features of the masked stimuli). The subjective visibility test at the last part of a trial was not included in the pilot test.

\subsection{Procedure}

The stimulus sequence in a trial was displayed in Figure 1: a fixation point was first displayed for $1500 \mathrm{~ms}$, then a tool picture appeared for $16 \mathrm{~ms}$ followed by a backward mask for $400 \mathrm{~ms}$. This mask was replaced by a blank screen for another $400 \mathrm{~ms}$, then two visible pictures of the picture forced-choice task or two visible names of the word forced-choice task appeared on the left and right of the fixation point respectively until a response was made. The sides on which the targets appeared were counterbalanced. Subjects were asked to judge which picture/word matched the masked tool picture presented earlier. In the picture forced-choice task, one of the two visible pictures was the same picture as the masked tool, the other was its mirror image. The subjects were asked to try to respond using the contour feature information of the masked stimuli if they had this information. In the word forced-choice task, one of the two names denoted the tool of the masked tool picture presented earlier and the other named a different tool. Finally, subjects reported on the quality of their subjective experience on a 4-point scale (1-the masked object completely unknown to the subject; 2-the masked object somewhat known; 3-the masked object almost known; 4-the masked object completely known).

Each subject completed 55 trials for one version of the forced-choice task. Before the forced-choice task began, subjects were informed that only the accuracy of response was important and that the probability of each of 


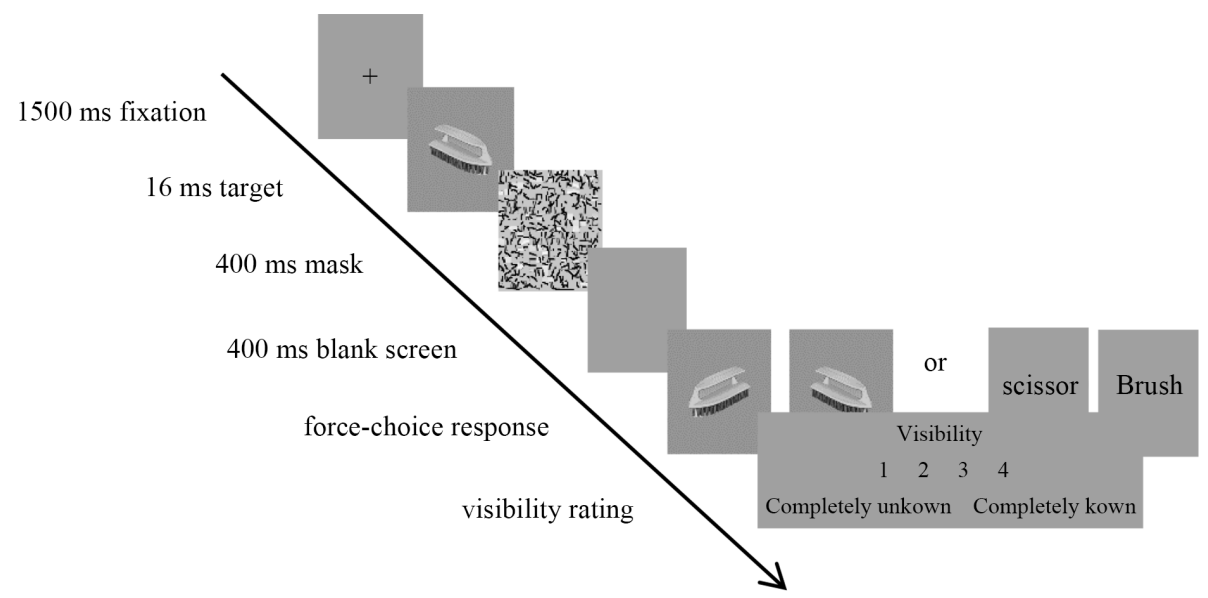

Figure 1. Stimulus sequence of a single trial in the experiment. Subjects were asked to judge which picture in the picture version forced-choice task or which word in the word version forced-choice task matched the masked tool picture presented earlier.

the two visible pictures or names representing the masked tool was equal. After the experiment, the subjects were asked to tell whether they could sense some features of the masked tool pictures (e.g. the contours) and whether they could tell what objects the masked tool pictures depicted. The combination of objective measure and visibility report were employed to reveal whether the subjects were indeed aware of the contours and orientations.

\section{Results}

\subsection{The Distribution of Visibility Rating}

In the word-version of the forced-choice task, the mean numbers of each rating per subject, the total number of each response summed over all subjects, and the ratio of the total number of each rating to the total number of all ratings for all subjects were $34,610,61.6 \%$ for rating " 1 "; $17,304,30.7 \%$ for rating " 2 "; 4, 73, 7.4\% for rating " 3 " and 0 , 3, 0.3\% for response " 4 ", respectively (Table 1). In the picture-version of the forced-choice task, the mean number of each rating per subject, the total number of each rating summed over all subjects, and the ratio of the total number of each rating to the total number of all ratings for all subjects were 27, 514, 49.2\% (for rating “1”); 16, 300, 28.7\% (for rating “2”); 10, 182, 17.4\% (for rating “3”) and 3, 49, 4.7\% (for rating “4”) (Table 1).

\subsection{Results of Analyses with Visibility Rating 4 Data Excluded}

All subjects reported they could sense some features of the masked stimuli in most trials but could seldom tell the identity of the masked tool pictures. In trials given a visibility 4 judgment (the masked tool completely known), it was likely that subjects could be aware of both the identity and the features of the masked stimuli. Since we were interested in examining partial awareness, we excluded these data in this analysis. There were nearly 30 trials where subjects judged the visibility as 1 (the masked tool completely unknown). We suspect that in those trials, although subjects claimed that they could not determine what the object was, they might have sensed some features of the masked tool. This possibility was supported by the finding of the pilot experiment where the mean number of trials in which subjects did not reported sensible features was $6 \pm 4$. Therefore, we first analyzed the data with trials judged as having visibility levels 1,2 and 3.

All subjects performed at the chance level in both the word and the picture recognition of the masked tool. In the word-version forced-choice task, the mean percentage of correct recognition was $50.61 \%$ which did not deviate from $50 \%$ significantly, $\mathrm{SD}=9.102 \%, \mathrm{t}(17)=0.285, p=0.779$. In the picture-version forced-choice task, the mean percentage of correct recognition was $51.00 \%$, SD $=9.062 \%$, again not significantly different from the chance level, $\mathrm{t}(18)=0.481, p=0.636$. Moreover, the d' values were not significantly different from zero in both forced choice tasks, $p s>0.624$. 
Table 1. The distribution of visibility rating.

\begin{tabular}{lcccc}
\hline & $\begin{array}{c}\text { Visibility } \\
\text { rating }\end{array}$ & $\begin{array}{c}\text { The mean numbers of each } \\
\text { rating per subject }\end{array}$ & $\begin{array}{c}\text { The total number of each } \\
\text { response summed over } \\
\text { all subjects }\end{array}$ & $\begin{array}{c}\text { The ratio of the total number of } \\
\text { each rating to the total number } \\
\text { of all ratings for all subjects }\end{array}$ \\
\hline Picture version & 27 & 16 & 514 & $49.2 \%$ \\
& 3 & 10 & 300 & $28.7 \%$ \\
\hline & 4 & 3 & 492 & $17.4 \%$ \\
\hline & 1 & 34 & 610 & $4.7 \%$ \\
\hline
\end{tabular}

\subsection{Results of Analyses with Visibility Ratings 1 and 4 Excluded}

Subjects judged the visibility of the masked object as 1 (completely unknown of the masked tool) in many trials which could contribute to the chance-level performance reported in the 3.2 result section. In order to include only the trials with the best chance of indicating partial awareness, we excluded both the trials judged as completely unknown (“1”) and completely known (“4”) from this analysis. Due to the exclusion of trials of visibility ratings of 1 and 4, some subjects had too few trials for further analysis. As a result, subjects with at least 20 trials of judged visibility ratings of 2 and 3 were included.

Again, all subjects performed at the chance level in both the word and picture recognition of the masked tool. In the word-version forced-choice task $(\mathrm{N}=12)$, the mean percentage of correct recognition was $54.25 \%$ which was not significantly different from the chance level, $\mathrm{SD}=12.211 \%, \mathrm{t}(11)=1.206, p=0.253$. In the picture-version forced-choice task $(\mathrm{N}=14)$, the mean percentage of correct recognition was $53.36 \%$, again not significantly different from the chance level, $\mathrm{SD}=10.895 \%, \mathrm{t}(13)=1.153, p=0.270$. Moreover, the $\mathrm{d}$ ' values were not significantly different from zero in both forced choice tasks, $p \mathrm{~s}>0.272$.

\subsection{Results of Analyses on Data with Complex and Simple Features}

Although the analyses in Sections 3.2 and 3.3 showed the recognition to be at the chance level, the extent of complexity of the masked stimuli could possibly lead to the chance level results. The subjects might be able to detect only simple features but not complex features. That is, in trials with masked complex stimuli, subjects might detect only a fraction of the stimuli and hence were not sure about the correct response. To examine this possibility, we selected only trials with masked simple stimuli such as a pen which had only a main oblique contour.

In order to maximize the chance of finding an above chance level performance, we analyzed only the picture-version of forced-choice task data. Twelve subjects with more than 12 trials of visibility ratings 2 and 3 (of which 9 subjects had at least 17 trials and 3 subjects had at least 12 trials met the criterion of selection) were included in the analysis. The results showed that these 12 subjects as a group performed at the chance level in the recognition of the masked tool. In the picture-version forced-choice task $(\mathrm{N}=12)$, the mean percentage of correct recognition was 52.25\%, not significantly different from the chance level, $\mathrm{SD}=12.389 \%, \mathrm{t}(11)=0.629, p=$ 0.542. Moreover, the d' value was not significantly different from zero, $\mathrm{t}(11)=0.662, p=0.522$.

\subsection{Analysis Results for Every Masked Picture}

In this analysis, the pictures that received visibility ratings of 2 or 3 were included. The results showed that the mean percentages of correct recognition were near 50\% for most masked pictures. However there were 6 trials in which the mean percentage of correct recognition were more than 75\%, and also 6 trials in which the mean percentage of correct recognition were less than 25\%. These results suggested that subjects in those cases either could see the contents of the picture accurately at an above chance level, or saw the wrong object at an above 
chance level.

\subsection{Results for Trials with Visibility 3 and 4}

As noted in section 3.2, in trials judged as having a visibility 4 (the masked tool completely known), it was likely that subjects could be aware both of the identity and the features of the masked stimuli. In the picture-version of the forced-choice task, two subjects gave visibility 4 judgment close to or above 10 times (one 9 and the other 14 times) out of 55 trials. However, their mean percentages of correct picture recognition for these trials were 33\% and $36 \%$, respectively, suggesting that they saw the wrong picture at an above chance level.

In addition, we can see from result section 3.1 that the number of trials judged as having visibility 4 was larger in the picture-version forced-choice task (49 times) as compared with the word-version forced-choice task (3 times). This was also the case with the visibility level 3 judgment, with 182 trials in the picture-version forcedchoice task versus 73 trials in the word-version forced-choice task.

\section{Discussion}

In the current study, we directly investigated whether the subjects could be aware of the contents of masked stimuli at the partial awareness level. Before the experiment, we speculated that the results of word-version forced-choice task would be at the chance level whereas the results of picture-version forced-choice task would be above the chance level due to partial awareness of features of the masked tool pictures but lack of information to determine the identity of the object. However, the results revealed that responses to the forced-choice tasks were all at the chance level except in the case of the analyses for every single picture being analyzed in the picture-version of the forced-choice task. The results are explained in the context of interaction hypothesis and level of processing hypothesis below.

The subjects responded with visibility level judgments of 2 and 3 in many cases to indicate that they could perceive some features of the masked stimuli indicating subjective partial awareness. However, the results of Sections 3.2 and 3.3 showed that the subjectively sensed features of the masked stimuli could be seldomly used helpfully for the forced-choice test which was supposed to measure the objective performance. All objective tests' performances were at the chance level. The chance performance might be due to these sensed features being scattered and incomplete, being only an insufficient part of the whole object to lead to correct responses in the forced-choice task. However, in the result Section 3.4, even when we selected the trials of simple stimuli (i.e. the stimuli having only a prominent oblique contour) with visibility ratings of 2 and 3 , the results still did not indicate that the subjects could make use of the subjectively perceived features correctly. We thought that these results supported the hypothesis of interaction between masked stimuli and the mask, according to which the masked stimuli and mask sequence was fused and perceived as one dynamic stimulus rather than two discrete, static ones (Sumner, 2007). In other words, the interaction between the masked stimulus and the mask could lead to the perception of a single stimulus. The idea of target-mask interaction was similar to the object updating hypothesis which posits a "process whereby recently sampled information is integrated with an existing representation of a scene, resulting in an updated version" of the percept (Lleras \& Enns, 2004). In our study, the contents of consciousness might be altered by the interaction between the masking stimulus and the masked target object picture.

Furthermore, the single picture analysis in result Section 3.5 showed that subjects could perceive the features of some pictures above the chance level while others below chance level. Thus, the results were much more complex than we expected. We think that the interaction between the masked target stimuli and the mask might have distorted the perceived feature contents in trials with below chance level performance, whereas some perceived features of the masked stimuli were robust enough to survive the making in the trials with above chance level performance. The latter finding supports the suggestion of correct perception of some target contents at a partial awareness level. We suggest that the impact of the interaction can be of three types, minimal, creating an altered percept, or mutually obliterating between the mask and the target. When it is minimal, some important features of the object can still be perceived; when it creates an altered percept, the two percepts are somewhat fused and the perceptible features are not the important ones and not enough to match it to the choices; when it is mutually obliterating, the two percepts are completely fused and no features of the object can be distinguished from the mask. The three results of at/above/below chance level performance were consistent with the partial awareness hypothesis which suggested a degraded nature of the stimulus caused by the interaction between the 
masking stimulus and a briefly displayed stimulus (Kouider et al., 2010). The results also suggested a continuous nature of the partial awareness state (Casali et al., 2013; Tu et al., 2015).

The results were also consistent with the level of processing hypothesis (Windey, Gevers \& Cleeremans, 2013), according to which the level of processing imposed by task requirements would influence the transition from unconscious to conscious perception. Specifically, the more complex the required processing is, the more discontinuous (or more discrete) the transition is from an unconscious to a conscious processing. Windey and colleagues (2013) have pointed out that in tasks involving relatively complex stimuli, such as number categorization (as opposed to physical feature matching), the transition from unconscious to conscious information processing is more discontinuous (Del Cul, Baillet, \& Dehaene, 2007) than in simple information processing such as tasks involving shape identification (Sandberg, Timmermans, Overgaard, \& Cleeremans, 2010). In our study, the visibility judgment showed that the number of trials given visibility 4 judgment was larger in the picture-version of the forced-choice task (49) than in the word-version of the task (3). This was also the case with the visibility level 3 judgment, with 182 trials in the picture-version forced-choice task versus 73 trials in the word-version forced-choice task (see result Sections 3.1 and 3.6). We hypothesize that the low, sensory level processing of the masked target stimulus provided more useful partial information (e.g., contours of the masked tools) for the picture-version of the forced-choice task than for the word-version of the task. This led to the more gradual visibility ratings in the picture than in the word version of the test.

The result Section 3.6 showed that the accuracy rates in the picture-version of the forced-choice task of two subjects whose visibility level 4 data were analyzed were rather low (33\% and $36 \%$, respectively). The low accuracy rate also supported the interaction hypothesis, because the subjects could have perceived obviously distorted features.

In summary, the results of current research are consistent with the interaction hypothesis and the level of processing hypothesis. However, we think that the form and complexity of the masking stimuli could affect the interaction effect leading to more or less correct responses in the forced-choice task. Future experiments should pay attention to the possible confusion by interaction between the masked stimulus and the mask in unconscious studies using masking paradigm.

\section{Acknowledgements}

This research was supported by the National Natural Science Foundation of China (31500871, 31400870, 31400901).

\section{References}

Arminjon, M., Preissmann, D., Chmetz, F., Duraku, A., Ansermet, F., \& Magistretti, P. (2015). Embodied Memory: Unconscious Smiling Modulates Emotional Evaluation of Episodic Memories. Frontiers in Psychology, 6, 650. http://dx.doi.org/10.3389/fpsyg.2015.00650

Atas, A., Vermeiren, A., \& Cleeremans, A. (2013). Repeating a Strongly Masked Stimulus Increases Priming and Awareness. Consciousness and Cognition, 22, 1422-1430. http://dx.doi.org/10.1016/j.concog.2013.09.011

Block, N. (2007). Consciousness, Accessibility, and the Mesh between Psychology and Neuroscience. Behavioral and Brain Sciences, 30, 481-499. http://dx.doi.org/10.1017/S0140525X07002786

Casali, A. G., Gosseries, O., Rosanova, M., Boly, M., Sarasso, S., Casali, K. R., et al. (2013). A Theoretically Based Index of Consciousness Independent of Sensory Processing and Behavior. Science Translational Medicine, 5, $198 \mathrm{ra} 105$. http://dx.doi.org/10.1126/scitranslmed.3006294

Del Cul, A., Baillet, S., \& Dehaene, S. (2007). Brain Dynamics Underlying the Nonlinear Threshold for Access to Consciousness. PLOS Biology, 5, e260. http://dx.doi.org/10.1371/journal.pbio.0050260

Faivre, N., \& Kouider, S. (2011). Multi-Feature Objects Elicit Nonconscious Priming Despite Crowding. Journal of Vision, 11, 1-10. http://dx.doi.org/10.1167/11.3.2

Heeks, F., \& Azzopardi, P. (2015). Thresholds for Detection and Awareness of Masked Facial Stimuli. Consciousness and Cognition, 32, 68-78. http://dx.doi.org/10.1016/j.concog.2014.09.009

Henson, R. N., Mouchlianitis, E., Matthews, W. J., \& Kouider, S. (2008). Electrophysiological Correlates of Masked Face Priming. NeuroImage, 40, 884-895. http://dx.doi.org/10.1016/j.neuroimage.2007.12.003

Kouider, S., \& Dupoux, E. (2004). Partial Awareness Creates the "Illusion” of Subliminal Semantic Priming. Psychological Science, 15, 75-81. http://dx.doi.org/10.1111/j.0963-7214.2004.01502001.x 
Kouider, S., de Gardelle, V., \& Dupoux, E. (2007). Partial Awareness and the Illusion of Phenomenal Consciousness. Behavioral and Brain Sciences, 30, 510-511. http://dx.doi.org/10.1017/S0140525X07002919

Kouider, S., de Gardelle, V., Sackur, J., \& Dupoux, E. (2010). How Rich Is Consciousness? The Partial Awareness Hypothesis. Trends in Cognitive Sciences, 14, 301-307. http://dx.doi.org/10.1016/j.tics.2010.04.006

Liu, C., Sun, Z., Jou, J., Martens, U., Yang, Q., Qiu, J., et al. (2015). Category-Selective Attention Modulates Unconscious Processing: Evidence from ERP. British Journal of Education, Society \& Behavioural Science, 7, 220-231. http://dx.doi.org/10.9734/BJESBS/2015/16565

Lleras, A., \& Enns, J. T. (2004). Negative Compatibility or Object Updating? A Cautionary Tale of Mask-Dependent Priming. Journal of Experimental Psychology: General, 133, 475-493. http://dx.doi.org/10.1037/0096-3445.133.4.475

Montoro, P. R., Luna, D., \& Ortells, J. J. (2014). Subliminal Gestalt Grouping: Evidence of Perceptual Grouping by Proximity and Similarity in Absence of Conscious Perception. Consciousness and Cognition, 25, 1-8. http://dx.doi.org/10.1016/j.concog.2014.01.004

Pinto, Y., van Gaal, S., de Lange, F. P., Lamme, V. A., \& Seth, A. K. (2015). Expectations Accelerate Entry of Visual Stimuli into Awareness. Journal of Vision, 15, 13. http://dx.doi.org/10.1167/15.8.13

Sandberg, K., Timmermans, B., Overgaard, M., \& Cleeremans, A. (2010). Measuring Consciousness: Is One Measure Better than the Other? Consciousness and Cognition, 19, 1069-1078. http://dx.doi.org/10.1016/j.concog.2009.12.013

Sumner, P. (2007). Negative and Positive Masked-Priming-Implications for Motor Inhibition. Advances in Cognitive Psychology, 3, 317-326. http://dx.doi.org/10.2478/v10053-008-0033-0

Tu, S., Jou, J., Cui, Q., Zhao, G., Wang, K., Hitchman, G., et al. (2015). Category-Selective Attention Interacts with Partial Awareness Processes in a Continuous Manner: An fMRI Study. Cogent Psychology, 21046243. http://dx.doi.org/10.1080/23311908.2015.1046243

Tu, S., Qiu, J., Martens, U., \& Zhang, Q. (2013). Category-Selective Attention Modulates Unconscious Processes in the Middle Occipital Gyrus. Consciousness and Cognition, 22, 479-485. http://dx.doi.org/10.1016/j.concog.2013.02.007

Verleger, R., Jaśkowski, P., Aydemir, A., Lubbe, R. H. V. D., \& Groen, M. (2004). Qualitative Differences between Conscious and Nonconscious Processing? On Inverse Priming Induced by Masked Arrows. Journal of Experimental PsychologyGeneral, 133, 494-515. http://dx.doi.org/10.1037/0096-3445.133.4.494

Vermeiren, A., \& Cleeremans, A. (2012). The Validity of d’Measures. PloS One, 7, e31595. http://dx.doi.org/10.1371/journal.pone.0031595

Windey, B., Gevers, W., \& Cleeremans, A. (2013). Subjective Visibility Depends on Level of Processing. Cognition, 129, 404-409. http://dx.doi.org/10.1016/j.cognition.2013.07.012

Yun, X., Li, W., Qiu, J., Jou, J., Wei, D., Tu, S., et al. (2011). Neural Mechanisms of Subliminal Priming for Traumatic Episodic Memory: An ERP Study. Neuroscience Letters, 498, 10-14. http://dx.doi.org/10.1016/j.neulet.2011.04.040 